\title{
Biometric Personal Identification Based on Chinese Handwriting Signature
}

\author{
Yongjian Zhao \\ Information Engineering Institute, Shandong University (Weihai), China \\ jian123cn@sdu.edu.cn
}

Keywords: Identification, Handwriting, wavelet, characteristic, transform

Abstract. Handwriting signature is an important approach for biometric personal identification. In this paper, a new method is provided to extract signature characteristic through wavelet transform. At first, a series of 4th order B-spline wavelet is constructed on the basis of B-spline function. After applying wavelet transform to each stroke, stable signature character is synthesized. The performance of derived method is demonstrated by computer simulations.

\section{Introduction}

Biometric personal identification is an important research area aiming at automatic identity recognition. Chinese handwriting signature is combined actions of psychology, physiology and optics [1]. It is well-known that handwriting is easy to obtain and different people have different handwritings. Therefore, Chinese handwriting signature has long been legally established and widely accepted as a useful biometric feature for reliable identity verification [2].

The principle of online Chinese signature identification can be described as follows. At first, the true signature sample set provided by specific person is collected to a pattern library. After inputting certain signature which needs to be identified, one can compare signature characteristic value with those in the pattern library so as to identify whether it is authentic or forged. Characteristic extraction is the kernel step in a Chinese signature identification system, which determines the property of whole process to a large extent [3].

Wavelet transform has good performance such as adaptive feature and mathematical microscope feature [4]. In this paper, a method is derived to extract signature characteristic through wavelet transform. The 4th order B-spline function has good properties to cope with curve and surface in computer vision fields [5]. At first, we construct a series of 4th order B-spline wavelet on the basis of B-spline function. Then wavelet transform, based on the 4th order B-spline wavelet, is applied to each stroke according to wavelet decomposition formula. Furthermore, signature characteristic values of strokes is synthesized through a series of new formulas. As the extracted characteristic values can reflect the total signature characteristics, a specific signature can be classified as either authentic or forged. Therefore, an identification method is derived correspondingly and its successful performance is demonstrated by computer simulations.

\section{B-spline Wavelet Bases}

Wavelet bases play an important role in the whole process of wavelet transform. It is more stable and accurate to construct wavelet bases according to specific application occasion. B-spline function has many desirable properties, such as recursion, local positive supported, multi-scale and the smallest compact supported [5]. There is a trend to use B-spline function to construct curve and surface in the field of computer graphics [7,8]. B-spline wavelet bases constructed by B-spline function have desirable properties.

The 1 th order B-spline function $\mathrm{N}_{1}(\mathrm{x})$ is feature function in interval $[0,1]$. For $\mathrm{m} \geqslant 2, \mathrm{~N}_{\mathrm{m}}$ is recursively defined as $N_{m}(x)=\int_{-\infty}^{+\infty} N_{m-1}(x-t) N_{1}(t) d t=\int_{0}^{1} N_{m-1}(x-t) d t$.

The $m$ th order B-spline $\mathrm{N}_{\mathrm{m}}$ satisfies the following properties: 
(1) $N_{m}(x)=\frac{1}{(m-1) !} \Delta^{m} x_{+}{ }^{m-1}=\frac{1}{(m-1) !} \sum_{k=0}^{m}(-1)^{k}\left(\begin{array}{c}m \\ k\end{array}\right)(x-k)_{+}{ }^{m-1}$

where $\Delta f(x)=f(x)-f(x-1), x_{+}=x \quad(x \geq 0), x_{+}=0 \quad(\mathrm{x}<0)$;

(2) $N_{m}(x)>0$ for $0<\mathrm{x}<\mathrm{m}$;

(3) $\sum_{k=-\infty}^{+\infty} N_{m}(x-k)=1$;

(4) $N_{m}(x)=\frac{x}{m-1} N_{m-1}(x)-\frac{m-x}{m-1} N_{m-1}(x-1)$;

(5) The Fourier transform of $N_{1}(x)$ is as $\hat{N}_{1}(\omega)=\frac{1-e^{i \omega}}{i \omega}=e^{-\frac{i \omega}{2}}\left[\frac{\sin (\omega / 2)}{\omega / 2}\right]$;

(6) The Fourier transform of $N_{m}(x)$ can be described as $\hat{N}_{m}(\omega)=\left(\frac{1-e^{i \omega}}{i \omega}\right)^{m}=e^{-\frac{i m \omega}{2}}\left[\frac{\sin (\omega / 2)}{\omega / 2}\right]^{m}$.

Based on the desirable properties of B-spline function, we construct a function as $\phi_{1}(x)=N_{m}(x+m / 2)$, whose Fourier transform is as $\hat{\phi}_{1}(\omega)=\left[\frac{\sin (\omega / 2)}{\omega / 2}\right]^{m}$.

Suppose that $e_{n}(\omega)=\sum_{k=-\infty}^{+\infty} \frac{1}{(\omega+2 k \pi)^{n+2}}$, one can derive that $e_{n}^{1}(\omega)=-(n+2) \sum_{k=-\infty}^{+\infty} \frac{1}{(\omega+2 k \pi)^{n+2+1}}=-(n+2) e_{n+1}(\omega)$.

In other words, $e_{n+1}(\omega)=-\frac{1}{n+2} e_{n}^{1}(\omega)$.

As $e_{0}(\omega)=\sum_{k=-\infty}^{+\infty} \frac{1}{(\omega+2 k \pi)^{2}}=\frac{1}{4 \sin ^{2}(\omega / 2)}$, one can deduce that

$\left\{\begin{array}{l}e_{n+1}(\omega)=-\frac{1}{n+2} e_{n}^{1}(\omega) \\ e_{0}(\omega)=\frac{1}{4 \sin ^{2}(\omega / 2)}\end{array}\right.$

and $\sum_{k=-\infty}^{+\infty}|\hat{\phi}(\omega+2 k \pi)|^{2}=2^{2 m} \sin ^{2 m}\left(\frac{m}{2}\right) e_{2(m-1)}(\omega)$. Therefore,

$\hat{\phi}(\omega)=\left[\frac{\sin (\omega / 2)}{\omega / 2}\right]^{m} \cdot \frac{1}{\sin ^{m}(\omega / 2)} \cdot \frac{1}{\sqrt{\sum_{k=-\infty}^{+\infty} \frac{1}{(\omega+2 k \pi)^{2 m}}}}=\frac{1}{\omega^{m}} \cdot \frac{1}{\sqrt{e_{2(m-1)}(\omega)}}$,

where function $\phi(x)$ satisfies the define of multi-scale: $\sum_{k=-\infty}^{+\infty}|\hat{\phi}(\omega+2 k \pi)|^{2}=1$.

As $\hat{\phi}(2 \omega)=H(\omega) \hat{\phi}(\omega)$, one can deduce that $H(\omega)=2^{-m} \sqrt{\frac{e_{2(m-1)}(\omega)}{e_{2(m-1)}(2 \omega)}}$. After setting $\mathrm{m}=4$, 4th B-spline wavelet bases can be constructed.

The discrete inverse Fourier of $\mathrm{H}(\omega)$ is impulse response $\left\{\mathrm{h}_{1}\right\} \mathrm{l}=0 \cdots 511$ from scaling function $\psi(\mathrm{t})$, whose value can be calculated. Due to space, we do not give the value of hl and $g_{1}(l=0 \cdots 511)$ where $g_{k}$ $=(-1)^{\mathrm{k}-1} \mathrm{~h}_{512-\mathrm{k}}, \mathrm{k}=1 \cdots 511$. 


\section{The Wavelet Presentation of Strokes}

After preprocessing, each stroke from specific signature can be expressed by 512 discrete complex numbers. Supposed that the signature have $M$ strokes, the mth stroke can be expressed as

$\left\{\mathrm{f}_{\mathrm{m}}(\mathrm{k})=\mathrm{x}_{\mathrm{m}}(\mathrm{k})+\mathrm{i} \mathrm{y}_{\mathrm{m}}(\mathrm{k}) \quad \mathrm{k}=0,1, \ldots, \mathrm{N}-1 \mathrm{~N}=512 \mathrm{~m}=0,1, \ldots, \mathrm{M}-1\right\}$.

Above stroke signals are finite, whose multi-resolution can be expressed as

$$
\begin{aligned}
& \mathrm{A}_{\mathrm{j}}^{\mathrm{d}} \mathrm{f}_{\mathrm{m}}(\mathrm{n})=\sum_{k=0}^{2^{j+1} N-1} \mathrm{~h}(\mathrm{l}) \mathrm{A}_{\mathrm{j}+1}{ }^{\mathrm{d}} \mathrm{f}_{\mathrm{m}}(\mathrm{k})=\sum_{k=0}^{2^{j+1} N-1} \mathrm{~h}(\mathrm{l}) \mathrm{A}_{\mathrm{j}+1}{ }^{\mathrm{d}} \mathrm{x}_{\mathrm{m}}(\mathrm{k})+\mathrm{i} \sum_{k=0}^{2^{j+1} N-1} \mathrm{~h}(\mathrm{l}) \mathrm{A}_{\mathrm{j}+1}{ }^{\mathrm{d}} \mathrm{y}_{\mathrm{m}}(\mathrm{k}), \\
& \mathrm{D}_{\mathrm{j}} \mathrm{d}_{\mathrm{m}}(\mathrm{n})=\sum_{k=0}^{2^{j+1} N-1} \mathrm{~g}(\mathrm{l}) \mathrm{A}_{\mathrm{j}+1}{ }^{\mathrm{d}} \mathrm{f}_{\mathrm{m}}(\mathrm{k})=\sum_{k=0}^{2^{j+1} N-1} \mathrm{~g}(\mathrm{l}) \mathrm{A}_{\mathrm{j}+1}{ }^{\mathrm{d}} \mathrm{x}_{\mathrm{m}}(\mathrm{k})+\mathrm{i} \sum_{k=0}^{2^{j+1}} \mathrm{~g}(\mathrm{l}) \mathrm{A}_{\mathrm{j}+1}{ }^{\mathrm{d}} \mathrm{y}_{\mathrm{m}}(\mathrm{k})
\end{aligned}
$$

where $1=[k-2(2 n+1)] \bmod \left(2^{j+1} N\right) n=0,1, \ldots, 2^{j} N-1 j=-1,-2 \ldots-J J>0$ and $\mathrm{A}_{0}{ }^{\mathrm{d}} \mathrm{f}_{\mathrm{m}}(\mathrm{K})=\mathrm{f}_{\mathrm{m}}(\mathrm{K})=\mathrm{x}_{\mathrm{m}}(\mathrm{K})+\mathrm{iy} \mathrm{m}(\mathrm{k}) \mathrm{k}=0,1, \ldots, \mathrm{N}-1$.

When decomposed to jth order layer, the sample number of $\operatorname{Ajdfm}(k)$ and $\operatorname{Djdfm}(k)$ is $2 \mathrm{jN}$, respectively where $\mathrm{j}<0$ and $\mathrm{k}=0,1, \ldots, 2 \mathrm{jN}$. If the stroke is decomposed to Jth layer, its wavelet representation can be expressed as

$\{\{$ A-Jdfm(k) where $\mathrm{k}=0 \ldots 2-\mathrm{JN}\},\{\operatorname{Djdfm}(\mathrm{k})$ where $\mathrm{j}=-\mathrm{J},-\mathrm{J}+1 \ldots-2,-1 \mathrm{k}=0 \ldots 2 \mathrm{jN}\}\}$.

\section{The Characteristic Synthesis of a Signature}

Based on the properties of discrete complex wavelet transform, we construct a series of characteristic extraction formulas. Each stroke of a signature can be expressed as

$\left\{\left\{\mathrm{A}_{-\mathrm{J}}{ }^{\mathrm{d}} \mathrm{f}_{\mathrm{m}}(\mathrm{k}) \mathrm{k}=0 \ldots 2^{-\mathrm{J}} \mathrm{N}\right\},\left\{\mathrm{D}_{\mathrm{j}}^{\mathrm{d}} \mathrm{f}_{\mathrm{m}}(\mathrm{k}) \mathrm{j}=-\mathrm{J},-\mathrm{J}+1 \ldots-2,-1 \mathrm{k}=0 \ldots 2^{\mathrm{J}} \mathrm{N}\right\}\right\}$

where $\mathrm{N}=512$ and $\mathrm{m}=0,1, \ldots, \mathrm{M}-1$.

$\mathrm{A}_{-\mathrm{J}}{ }^{\mathrm{d}} \mathrm{f}_{\mathrm{m}}(\mathrm{k})$ can be described $\mathrm{A}_{-\mathrm{J}}{ }^{\mathrm{d}} \mathrm{f}_{\mathrm{m}}(\mathrm{k})=\mathrm{A}_{-\mathrm{J}}{ }^{\mathrm{d}} \mathrm{x}_{\mathrm{m}}(\mathrm{k})+\mathrm{i} \mathrm{A}_{-\mathrm{J}}{ }^{\mathrm{d}} \mathrm{y}_{\mathrm{m}}(\mathrm{k})$ where $\mathrm{k}=0,1 \ldots, 2^{-\mathrm{J}}$ and $\mathrm{m}=0,1, \ldots, \mathrm{M}-1$.

$D_{j}{ }^{d} f_{m}(k)$ can be described as $D_{j}{ }^{d} f_{m}(k)=D_{j}^{d} x_{m}(k)+i D_{j}^{d} y_{m}(k) \quad$ where $j=-J \ldots-1, \quad k=0,1 \ldots, 2^{j} N$ and $\mathrm{m}=0,1, \ldots, \mathrm{M}-1$.

For $\mathrm{A}_{-\mathrm{J}}{ }^{\mathrm{d}} \mathrm{f}_{\mathrm{m}}(\mathrm{k})$ and $\mathrm{D}_{\mathrm{j}}^{\mathrm{d}} \mathrm{f}_{\mathrm{m}}(\mathrm{k})$, we set

$$
\begin{aligned}
& \mathrm{A}_{-{ }_{\mathrm{J}}}^{\mathrm{d}} \overline{x_{m}}=\frac{1}{2^{-J} N} \sum_{K=0}^{2^{-J}} A_{-J}{ }^{d} x_{m}(k), \quad \mathrm{A}_{-\mathrm{J}}^{\mathrm{d}} \overline{y_{m}}=\frac{1}{2^{-J} N} \sum_{K=0}^{2^{-J}} A_{-J}{ }^{d} y_{m}(k), \\
& \mathrm{D}_{\mathrm{j}}^{\mathrm{d}} \overline{x_{m}}=\frac{1}{2^{-J} N} \sum_{K=0}^{2^{-J}} D_{j}{ }^{d} x_{m}(k) \text { and } \mathrm{D}_{\mathrm{j}}^{\mathrm{d}} \overline{y_{m}}=\frac{1}{2^{-J} N} \sum_{K=0}^{2^{-J}} D_{j}{ }^{d} y_{m}(k) .
\end{aligned}
$$

For $F A_{-J}^{d} f_{m}$ and $F D_{j}^{d} f_{m}$, we set

$$
\begin{aligned}
& F A_{-J}^{d} f_{m}=\frac{\sqrt{\sum_{K=0}^{2^{-J}}\left(\left(A_{-J}{ }^{d} x_{m}(k)-A_{-J}{ }^{d} \overline{x_{m}}\right)^{2}+\left(A_{-J}{ }^{d} y_{m}(k)-A_{-J}{ }^{d} \overline{y_{m}}\right)^{2}\right.}}{\sum_{m=0}^{M-1} \sqrt{\sum_{K=1}^{2^{-J} N-1}\left(\left(A_{-J}{ }^{d} x_{m}(k)-A_{-J}{ }^{d} x_{m}(k-1)\right)^{2}+\left(A_{-J}{ }^{d} y_{m}(k)-A_{-J}{ }^{d} y_{m}(k-1)\right)^{2}\right.}}, \\
& F D_{j}^{d} f_{m}=\frac{\sqrt{\sum_{K=0}^{2^{-J}}\left(\left(D_{j}{ }^{d} x_{m}(k)-D_{j}{ }^{d} \overline{x_{m}}\right)^{2}+\left(D_{j}{ }^{d} y_{m}(k)-D_{j}{ }^{d} \overline{y_{m}}\right)^{2}\right.}}{\sum_{m=0}^{M-1} \sqrt{\sum_{K=1}^{2^{-J}\left(\left(D_{j}{ }^{d} x_{m}(k)-D_{j}{ }^{d} x_{m}(k-1)\right)^{2}+\left(D_{j}{ }^{d} y_{m}(k)-D_{j}{ }^{d} y_{m}(k-1)\right)^{2}\right.}}}
\end{aligned}
$$

where $\mathrm{j}=-\mathrm{J},-\mathrm{J}+1, \ldots,-1$ and $\mathrm{m}=0,1, \ldots, \mathrm{M}-1$.

Above formulas have desirable performances such as rotation invariance, transformation invariance and scale invariance. The characteristics of mth stroke are $\left\{F A_{-J}{ }^{d} f_{m}, F D_{j}^{d} f_{m}\right.$ where $\mathrm{j}=-\mathrm{J},-\mathrm{J}+1, \ldots,-1\}$, whose various layer characteristic number $\mathrm{J}+1$. In our experiments, we set $\mathrm{J}=5$ and 
demonstrate by experiments that $\left\{F A_{-5}{ }^{d} f_{m}, F D_{j}{ }^{d} f_{m} \mathrm{j}=-5,-4,-3\right\}$ are robust and reliable. If we choose $\left\{F A_{-5}^{d} f_{m}, F D_{j}^{d} f_{m} \mathrm{j}=-5,-4,-3\right\}$ as characteristic of $\mathrm{mth}$ stroke, the whole signature characteristic can be expressed as $\left\{F A_{-5}{ }^{d} f_{m}, F D_{j}^{d} f_{m} \quad \mathrm{j}=-5,-4,-3 \mathrm{~m}=0,1, \ldots, \mathrm{M}-1\right\}$.

\section{Experiment Results}

In our experiments, 20 signatures from a genuine writer are collected on a computer connected digitizer. The method derived in this paper is utilized to extract signature characteristic and construct a pattern library. The other 2 signatures from genuine writer and forgery writer are also collected and applied characteristic extraction, respectively. At last, we use classical distance match method such as Euclidean distance and Mahalanobis [7,8] distance and get identification results. Typical results are shown in Table 1.

Table 1 Comparisons of the identification results

\begin{tabular}{|c|c|c|}
\hline method & mean identification rate(\%) & optimum identification rate(\%) \\
\hline method in this paper & 88.5 & 93.4 \\
\hline method in [6] & 84.2 & 86.3 \\
\hline
\end{tabular}

\section{Conclusions}

By verifying the writer's identity on the basis of handwriting, Chinese signature identification can accurately distinguish various pseudo signatures from the real one. Therefore, this technique has become an active research topic in the fields of computer vision and pattern recognition. At present, biometric personal identification based on handwriting signature has wide used in credit card transaction and the authority of electronic documents.

Based on wavelet transform, a new signature identification method is presented in this paper. This method develops the 4th B-spline wavelet bases and applies wavelet transform to each stroke of a signature. A stable and effective signature pattern library is formed and a proper classifier is developed, thus enhancing the effects of identification. The simulation results initiate a potential application to treat Chinese signature identification problem.

\section{References}

[1] Zhaoqi Bian, Pattern recognition, the Tsinghua University Press, 1994,120-138.

[2] Zhu Yong, Writer identification based on texture analysis, Acta automatica Sinica, 2005:231-247.

[3] Plamond R, Lorette G, Automatic signature verification and writer identification-the state of art, Pattern Recognition, 2005,32 (2):108-135.

[4] Peter N Belhumeur, Eigenfaces vs fisherfaces: recognition using class specific linear projection, IEEE Trans on Pattern Anal Machine Intelligent, 1997, 19 (7).

[5] Bovik A C, Gopal N, Emmoth T, Localized measurement of emergent image frequencies by Gabor wavelets, IEEE Trans. Inf. Theory, 2004, 56 (2):691-712.

[6] B D Ripley, Pattern recognition and neural networks, Cambrige : Cambrige University Press, 1996.

[7] Donohn D L, Denoising by soft threshold, IEEE Trans on Information Theory, 1995, 41 (5):613-627.

[8] Said H E S, Baker K D, Tan T N, Personal identification based on handwriting, In:Proc. 14th IAPR Inter. Conf. Pattern recognition,1998, 1761-1764. 